\title{
ONE-REGULAR CUBIC GRAPHS OF ORDER A SMALL NUMBER TIMES A PRIME OR A PRIME SQUARE
}

\author{
YAN-QUAN FENG and JIN HO KWAK
}

(Received 26 July 2001; revised 6 March 2003)

Communicated by B. D. McKay

\begin{abstract}
A graph is one-regular if its automorphism group acts regularly on the set of its arcs. In this paper we show that there exists a one-regular cubic graph of order $2 p$ or $2 p^{2}$ where $p$ is a prime if and only if 3 is a divisor of $p-1$ and the graph has order greater than 25. All of those one-regular cubic graphs are Cayley graphs on dihedral groups and there is only one such graph for each fixed order. Surprisingly, it can be shown that there is no one-regular cubic graph of order $4 p$ or $4 p^{2}$.
\end{abstract}

2000 Mathematics subject classification: primary 05C25, $20 \mathrm{~B} 25$.

Keywords and phrases: Cayley graph, arc-transitive graph, one-regular graph.

\section{Introduction}

Throughout this paper a graph means an undirected finite one, without loops or multiple edges. For a graph $X$, we denote by $V(X), E(X)$ and $\operatorname{Aut}(X)$ its vertex set, its edge set and its automorphism group, respectively. For further group- and graph-theoretic notation and terminology, we refer the reader to [12] and [13].

Let $G$ be a finite group and $S$ a subset of $G$ such that $1 \notin S$ and $S=S^{-1}$. The Cayley graph $X=\operatorname{Cay}(G, S)$ on $G$ with respect to $S$ is defined to have vertex set

$$
V(X)=G
$$

and edge set

$$
E(X)=\{(g, s g) \mid g \in G, s \in S\} .
$$

Supported by EYTP, SRF for ROCS in China and $\mathrm{Com}^{2} \mathrm{MaC}-\mathrm{KOSEF}$ in Korea.

(C) 2004 Australian Mathematical Society 1446-7887/04 \$A2.00+0.00 
From the definition, $\operatorname{Cay}(G, S)$ is connected if and only if $S$ generates the group $G$.

A permutation group $G$ on a set $\Omega$ is said to be semiregular if for each $\alpha \in \Omega$, the stabilizer $G_{\alpha}$ of $\alpha$ in $G$ is the identity group, and regular if it is semiregular and transitive. Let $X$ be a graph. A subgroup $G$ of $\operatorname{Aut}(X)$ is said to be regular and one-regular if it acts regularly on the vertex set and the arc set of $X$, respectively. A graph $X$ is said to be vertex-transitive, edge-transitive, arc-transitive and one-regular (or arc-regular) if $\operatorname{Aut}(X)$ is vertex-transitive, edge-transitive, arc-transitive and oneregular, respectively, and half-transitive if $\operatorname{Aut}(X)$ is vertex-transitive, edge-transitive, but not arc-transitive.

Clearly, a one-regular graph of regular valency must be connected and a graph of valency 2 is one-regular if and only if it is a cycle. Marušič [17] and Malnič et al. [15] constructed two different kinds of infinite families of one-regular graphs of valency 4, and Xu [24] gave a classification of one-regular circulant graphs of valency 4. One-regular cubic graphs have also received considerable attention. The first example of one-regular cubic graph was constructed by Frucht [9] with 432 vertices, and lots of work has been done on one-regular cubic graphs as part of a more general problem dealing with the investigation of a class of arc-transitive cubic graphs (see [4, 6, 20]). In 1997, Marušič and Xu [19] showed a way to construct a one-regular cubic graph $Y$ from a half-transitive graph $X$ of valency 4 with girth 3 by letting the triangles of $X$ be the vertices in $Y$ with two triangles being adjacent in $Y$ when they share a common vertex in $X$. Thus, one can construct infinitely many one-regular cubic graphs from the infinite family of half-transitive graphs of valency 4 with girth 3 constructed by Alspach $e t$ al. in [1] and from another infinite family of half-transitive graphs constructed by Marušič and Nedela in [18]. Recently, Feng et al. [8] classified one-regular cubic Cayley graphs on abelian or dihedral groups. In this paper, we classify one-regular cubic graphs of order $2 p, 4 p, 2 p^{2}$ or $4 p^{2}$, where $p$ is a prime. A one-regular cubic graph of order $2 p$ or $2 p^{2}$ is a Cayley graph on a dihedral group. Such a graph exists only when 3 is a divisor of $p-1$ and the graph has order greater than 25 , and it is unique for each fixed order. Thus there exists a unique one-regular cubic Cayley graph on the dihedral group of order 26, which is the least one-regular cubic graph by Conder and Dobcsányi [3]. Surprisingly, there is no one-regular cubic graph of order $4 p$ or $4 p^{2}$.

We know that Cheng and Oxley [2] classified arc-transitive graphs of order $2 p$. Among the graphs in their classification, there is a unique one-regular cubic graph for each prime $p \geq 13$ such that 3 is a divisor of $p-1$. In this paper we show that a oneregular cubic graph of order twice an odd integer is a Cayley graph (Corollary 3.3), which implies that the unique one-regular cubic graph of a fixed order $2 p$ in [2] must be a Cayley graph on a dihedral group. By using Corollary 3.3 we classify one-regular cubic graphs of order $2 p^{2}$ and the same method can be used to classify one-regular cubic graphs of some orders, such as $6 p, 6 p^{2}, 2 p^{3}, 6 p^{3}$. Note that it is easy to 
classify one-regular cubic graphs of order $3 p, 3 p^{2}, 5 p, 5 p^{2}$, etc. since the valency 3 forces $p=2$.

\section{Preliminaries}

We start with introducing five propositions for later applications in this paper. The first one has achieved a sort of folklore status, whereas the others are well known as group-theoretic results.

PROPOSITION 2.1. A graph $X$ is a Cayley graph if and only if Aut $(X)$ contains a regular subgroup.

PROPOSITION 2.2 ([23, Proposition 4.4]). Any abelian transitive permutation group on a set is regular.

PROPOSITION 2.3 ([23, Theorem 3.4]). Let $G$ be a permutation group on $\Omega$ and $\alpha \in \Omega$. Denote by $\alpha^{G}$ the orbit of $\alpha$ under $G$. Let $p$ be a prime number and let $p^{m}$ be a divisor of $\left|\alpha^{G}\right|$. Then $p^{m}$ is also a divisor of $\left|\alpha^{P}\right|$ for any Sylow $p$-subgroup $P$ of $G$.

Let $\pi$ be a nonempty set of primes and $\pi^{\prime}$ the set of primes which are not in $\pi$. A finite group $G$ is called a $\pi$-group, if every prime factor of $|G|$ is in the set $\pi$. In this case, we also say that $|G|$ is a $\pi$-number.

Let $G$ be a finite group. A $\pi$-subgroup $H$ of $G$ such that $|G: H|$ is a $\pi^{\prime}$-number is called a Hall $\pi$-subgroup of $G$.

The following proposition is due to Hall [22].

PROPOSITION 2.4 ([22, Theorem 9.1.7]). If $G$ is a finite solvable group, then every $\pi$-subgroup is contained in a Hall $\pi$-subgroup of $G$. Moreover, all Hall $\pi$-subgroups of $G$ are conjugate.

Let $p$ be a prime. A finite group $G$ is called a $p$-group if it is a $\pi$-group for $\pi=\{p\}$.

PROPOSITION 2.5 ([13, Theorem 7.2]). Let $N$ be a nontrivial normal subgroup of a p-group $G$ and $Z(G)$ the center of $G$. Then $N \cap Z(G) \neq 1$.

The next two propositions give a classification of one-regular cubic Cayley graphs on abelian or dihedral groups.

PROPOSITION 2.6 ([8, Theorem 3.1]). There is no one-regular cubic Cayley graph on an abelian group. 
PROPOSITION 2.7 ([8, Theorem 4.1]). A cubic Cayley graph $X$ on a dihedral group is one-regular if and only if $X$ is isomorphic to $\operatorname{Cay}\left(D_{2 n},\left\{a, a b, a b^{-k}\right\}\right)$ for $n \geq 13$, $3 \leq k<n$, and $k^{2}+k+1 \equiv 0(\bmod n)$, where $D_{2 n}=\langle a, b| a^{2}=b^{n}=1, a b a=$ $\left.b^{-1}\right\rangle$.

By checking Conder and Dobcsányi's list [3] of arc-transitive cubic graphs up to 768 vertices, we have the following proposition.

PROPOSITION 2.8. For any one-regular cubic graph $X,|V(X)| \geq 26$ and $|V(X)| \neq$ $4 p$ or $4 p^{2}$ for a prime $p \leq 13$.

\section{One-regular cubic graphs of order $2 p$ or $2 p^{2}$}

In this section we classify one-regular cubic graphs of order $2 p$ or $2 p^{2}$, where $p$ is a prime. Let $K_{3,3}$ be the bipartite graph of order 6 . It is well-known that $\operatorname{Aut}\left(K_{3,3}\right) \cong\left(S_{3} \times S_{3}\right) \rtimes \mathbb{Z}_{2}$ and so $\operatorname{Aut}\left(K_{3,3}\right)$ has a normal Sylow 3-subgroup. From this, one may easily show the following lemma.

LEMMA 3.1. Let $G$ be a vertex-transitive automorphism group of the graph $K_{3,3}$. If $|G|=18$ then $G$ has a regular subgroup of order 6 and its Sylow 3-subgroup contains a minimal normal subgroup of $G$ isomorphic to $\mathbb{Z}_{3}$.

LEMMA 3.2. A solvable one-regular automorphism group of a connected cubic graph contains a regular subgroup.

Proof. Suppose to the contrary; let $X$ be a counterexample of least order, that is, $X$ is of the smallest order with the following properties: $X$ is a connected cubic graph and its automorphism group $\operatorname{Aut}(X)$ contains a solvable one-regular subgroup $G$, which has no regular subgroup.

Let $N$ be a minimal normal subgroup of $G$. Since $G$ is solvable $N$ is elementary abelian, say $N=\mathbb{Z}_{p} \times \mathbb{Z}_{p} \times \cdots \times \mathbb{Z}_{p}=\mathbb{Z}_{p}^{m}$, for a prime $p$ and a positive integer $m$. By Proposition 2.2, $N$ cannot be transitive on $V(X)$. Denote by $\Sigma=\left\{B_{0}, B_{1}, \ldots, B_{l-1}\right\}$ the set of orbits of $N$ on $V(X)$. Since $N \triangleleft G, \Sigma$ is a complete block system of $G$. Consider the quotient graph $\bar{X}$ of $X$ defined by $V(\bar{X})=\Sigma$ and $\left(B_{i}, B_{j}\right) \in E(\bar{X})$ if and only if there exist $v_{i} \in B_{i}$ and $v_{j} \in B_{j}$ such that $\left(v_{i}, v_{j}\right) \in E(X)$. If $N$ has more than two orbits, Lorimer [14, Theorem 9] showed that $\bar{X}$ is a cubic graph and $G / N$ is a solvable one-regular subgroup of $\operatorname{Aut}(\bar{X})$ (also see [21]). The minimality of $X$ implies that $G / N$ has a regular subgroup, say $H / N$ on $V(\bar{X})$ and so $H$ acts regularly on $V(X)$, a contradiction. Thus we may assume that $N$ has only two orbits; $\Sigma=\left\{B_{0}, B_{1}\right\}$. Let $K$ be the subgroup of $G$ which fixes $B_{0}$ setwise and let $u \in B_{0}$. 
It follows that $G / K \cong \mathbb{Z}_{2}$ and the one-regularity of $G$ implies $G_{u} \cong \mathbb{Z}_{3}$, where $G_{u}$ is the stabilizer of $u$ in $G$. We also denote by $K_{u}$ and $N_{u}$ the stabilizers of $u$ in $K$ and $N$, respectively. Then $G_{u} \leq K, G_{u}=K_{u}$, and $K=N K_{u}=N G_{u}$. If $N$ is not semiregular, $N_{u} \cong \mathbb{Z}_{3}$. Since $N$ is abelian $N_{u}$ fixes $B_{0}$ pointwise. This implies $X \cong K_{3,3}$, the complete bipartite graph of order 6 , and consequently $N \cong \mathbb{Z}_{3} \times \mathbb{Z}_{3}$, which is impossible since $N$ is not minimal in $G$ by Lemma 3.1. If $N$ is semiregular then $|K|=|N|\left|G_{u}\right|=3 p^{m}$ and $|G|=6 p^{m}$. Now we consider three cases: $p=2$, $p=3$ or $p \neq 2,3$.

Case I: $p=2$. In this case $|G|=2^{m+1} \cdot 3$ and $|V(X)|=2^{m+1}$. By Proposition 2.3, each Sylow 2-subgroup of $G$ is transitive on $V(X)$ and so is regular because both the Sylow 2-subgroup and the graph $X$ have the same order $2^{m+1}$. It is impossible.

Case II: $p \neq 2,3$. In this case $|G|=2 \cdot 3 \cdot p^{m}$ and $|V(X)|=2 p^{m}$. Let $\pi=\{2, p\}$. By Proposition 2.4, $G$ has a Hall $\pi$-subgroup, say $H$. Then $|H|=2 p^{m}$. Since $G_{u} \cong \mathbb{Z}_{3}$ and $|H|$ has no divisor 3 , we have $H_{u}=1$, where $H_{u}$ is the stabilizer of $u$ in $H$. Thus $H$ has an orbit of length $2 p^{m}$ and so acts regularly on $V(X)$, a contradiction.

Case III: $p=3$. In this case $|G|=2 \cdot 3^{m+1}$ and $|V(X)|=2 \cdot 3^{m}$. It is easy to see that $K$ is the unique Sylow 3-subgroup of $G$. Therefore $Z(K) \neq 1$ (a nilpotent group has a non-trivial center) and $Z(K) \triangleleft \varangle$, that is $Z(K)$ is a characteristic subgroup of $K$. Thus $Z(K) \triangleleft G$. By Proposition 2.5 we have $N \cap Z(K) \neq 1$, and since $N \triangleleft G$ and $Z(K) \triangleleft G, N \cap Z(K) \triangleleft G$. By the minimality of $N, N \cap Z(K)=N$, which forces $N \leq Z(K)$. Let $u, v \in B_{0}$. Then $N \leq Z(K)$ implies $K_{u}=K_{v}$. It follows that $K_{u}$ fixes $B_{0}$ pointwise and so $X \cong K_{3,3}$. By Lemma $3.1 G$ has a regular subgroup, a contradiction.

Assume that $X$ is a one-regular cubic graph and let $A=\operatorname{Aut}(X)$. If $X$ has order $2 n$ with $n$ an odd integer, then $|A|=2 \cdot 3 \cdot n$. Since a group of order twice an odd integer is solvable, $A$ is solvable. By Lemma 3.2 and Proposition 2.1 we have the following corollary.

COROLLARY 3.3. A one-regular cubic graph of order twice an odd integer is a Cayley graph.

REMARK. Fang et al. [7] proved that Lemma 3.2 is also true for a connected graph of any prime valency.

Let $\mathbb{Z}_{n}=\{0,1,2, \ldots, n-1\}$ be the cyclic group of order $n$ written additively and let $\mathbb{Z}_{n}^{*}$ be the multiplication group of $\mathbb{Z}_{n}$ consisting of numbers in $\mathbb{Z}_{n}$ coprime to $n$. Then $\mathbb{Z}_{p^{m}}^{*} \cong \mathbb{Z}_{(p-1) p^{m-1}}$ for any odd prime $p$ and any positive integer $m$. If 3 is a divisor of $p-1$ then $\mathbb{Z}_{p^{m}}^{*}$ has a unique subgroup of order 3 . The proof of the following lemma is easy and we omit it. 
LEMMA 3.4. Let $p>3$ be a prime and $n=p$ or $p^{2}$. Then there exists an integer $1 \leq k<n$ such that $k^{2}+k+1 \equiv 0(\bmod n)$ if and only if $k$ is an element of order 3 in $\mathbb{Z}_{n}^{*}$.

THEOREM 3.5. Let $n=p$ or $p^{2}$ for a prime $p$. Then there exists a one-regular cubic graph $X$ of order $2 n$ if and only if 3 is a divisor of $p-1$ and $|V(X)| \geq 26$. Furthermore, for each prime $p$ with 3 being a divisor of $p-1$ and $n \geq 13$, there exists $a$ unique one-regular cubic graph $X$ of order $2 n$ and $X=\operatorname{Cay}(G, S)$, where $G=\left\langle a, b \mid a^{2}=b^{n}=1, a b a=b^{-1}\right\rangle$ is a dihedral group and $S=\left\{a, a b, a b^{-k}\right\}$ with $k$ being an element of order 3 in $\mathbb{Z}_{n}^{*}$.

ProOF. Let $X$ be a one-regular cubic graph of order $2 n$ where $n=p$ or $p^{2}$, and let $A=\operatorname{Aut}(X)$. By Proposition $2.8, p>3$ and by Corollary $3.3 X$ is a Cayley graph, say $X=\operatorname{Cay}(G, S)$, where $G$ is a group of order $2 n$. Thus, Proposition 2.6 implies that $G$ is nonabelian. Let $A_{1}$ denote the stabilizer of 1 in $A$ and $\operatorname{Aut}(G, S)=$ $\left\{\alpha \in \operatorname{Aut}(G) \mid S^{\alpha}=S\right\}$. Then $A_{1} \cong \mathbb{Z}_{3}$ and $\operatorname{Aut}(G, S) \leq A_{1}$. Since $X$ is connected, $\langle S\rangle=G$. We claim that $G$ is dihedral. But, it is obvious for $|G|=2 p$ because $G$ is nonabelian.

Assume that $|G|=2 p^{2}$. From an elementary group theory we know that up to isomorphism there are three nonabelian groups of order $2 p^{2}$ defined as:

$$
\begin{aligned}
& G_{1}(p)=\left\langle a, b \mid a^{2}=b^{p^{2}}=1, a b a=b^{-1}\right\rangle ; \\
& G_{2}(p)=\left\langle a, b, c \mid a^{p}=b^{p}=c^{2}=[a, b]=1, c^{-1} a c=a^{-1}, c^{-1} b c=b^{-1}\right\rangle ; \\
& G_{3}(p)=\left\langle a, b, c \mid a^{p}=b^{p}=c^{2}=1,[a, b]=[a, c]=1, c^{-1} b c=b^{-1}\right\rangle .
\end{aligned}
$$

Suppose to the contrary that $G \neq G_{1}(p)$. Let $x, y \in G_{2}(p)$ with $o(x)=2$ and $o(y)=p$. It is easy to show that $\langle x, y\rangle$ has order $2 p$, and hence $\langle x, y\rangle \neq G_{2}(p)$. Thus if $G=G_{2}(p)$ then $S$ consists of three involutions of $G_{2}(p)$ since $G=G_{2}(p)$ has no element of order $2 p$. Let $z \in G_{2}(p)$ be an element of order $p$ which is not in $\langle y\rangle$. Then $x, y$ and $z$ satisfy the same relations as do $c, a$ and $b$, and hence there is an automorphism of $G_{2}(p)$ mapping $x, y$ and $z$ to $c, a$ and $b$, respectively. Thus we may assume that $S=\{c, c a, c b\}$ because $\langle S\rangle=G$, and since the automorphism of $G_{2}(p)$ induced by $c \rightarrow c, a \rightarrow b$ and $b \rightarrow a$, interchanges $c a$ and $c b$, and fixes $c$, $|\operatorname{Aut}(G, S)|$ has a divisor 2. By $\operatorname{Aut}(G, S) \leq A_{1},\left|A_{1}\right|$ has a divisor 2, contrary to the fact that $A_{1} \cong \mathbb{Z}_{3}$. If $G=G_{3}(p)$ then $S$ consists of one involution, one element of order $p$ or $2 p$ and its inverse because all involutions of $G_{3}(p)$ can't generate $G_{3}(p)$. Since the automorphism group of $G_{3}(p)$ is transitive on the set of involutions of $G_{3}(p)$, we may assume that $S=\left\{c, a^{i} b^{j},\left(a^{i} b^{j}\right)^{-1}\right\}$ or $\left\{c, c a^{i} b^{j},\left(c a^{i} b^{j}\right)^{-1}\right\}$, where $a^{i} \neq 1$ and $b^{j} \neq 1$ since $\langle S\rangle=G$. The mapping $c \rightarrow c, a \rightarrow a^{i}$ and $b \rightarrow b^{j}$ induces an automorphism of $G_{3}(p)$, and so we may assume that $S=\left\{c, a b, a^{-1} b^{-1}\right\}$ 
or $\left\{c, c a b, c a^{-1} b\right\}$. For $S=\left\{c, a b, a^{-1} b^{-1}\right\}, X$ has a cycle of length $p$ passing through 1 and $a b$ but there exists no such cycle passing through 1 and $c$, contrary to the arc-transitivity of $X$. For $S=\left\{c, c a b, c a^{-1} b\right\}$, let $\alpha$ be a permutation on $G=G_{3}(p)$ defined by $\left(a^{i} b^{j} c^{k}\right)^{\alpha}=a^{-i} b^{j} c^{k}$ where $i, j$ and $k$ are integers. Let $g \in G$ and denote by $N(g)$ the neighborhood of $g$ in $X$. Now it is easy to check that $N\left(\left(a^{i} b^{j} c^{k}\right)^{\alpha}\right)=\left(N\left(a^{i} b^{j} c^{k}\right)\right)^{\alpha}$, implying that $\alpha$ is an automorphism of $X$. Since $\alpha$ fixes 1, we have $\alpha \in A_{1}$ and so $\left|A_{1}\right|$ has a divisor 2, a contradiction.

So far, we have proved that $X$ is a Cayley graph on a dihedral group. By Lemma 3.4 and Proposition 2.7 we have $|V(X)| \geq 26$ and $X=\operatorname{Cay}(G, S)$, where $G=\langle a, b|$ $\left.a^{2}=b^{n}=1, a b a=b^{-1}\right\rangle$ and $S=\left\{a, a b, a b^{-k}\right\}$ with $k$ being an element of order 3 in $\mathbb{Z}_{n}^{*}$. Note that $\mathbb{Z}_{n}^{*}$ has elements of order 3 if and only if 3 is a divisor of $p-1$. To prove Theorem 3.5 , we only need to prove the uniqueness of one-regular cubic graph of order $2 n$ when $p-1$ has a divisor 3 and $n \geq 13$. Since $\mathbb{Z}_{n}^{*}$ has only two elements of order 3 , that is $k$ and $k^{2}$, it suffices to prove that $\operatorname{Cay}\left(G,\left\{a, a b, a b^{-k}\right\}\right) \cong$ $\operatorname{Cay}\left(G,\left\{a, a b, a b^{-k^{2}}\right\}\right)$, which follows from the fact that the automorphism of $G$ induced by $a \rightarrow a$ and $b \rightarrow b^{-k^{2}}$ maps $\left\{a, a b, a b^{-k}\right\}$ to $\left\{a, a b, a b^{-k^{2}}\right\}$.

\section{No one-regular cubic graphs of order $4 p$ or $4 p^{2}$}

To show the non-existence of one-regular cubic graphs of order $4 p$ or $4 p^{2}$, we need to consider regular coverings of the complete graph $K_{4}$ of order 4 .

A graph $\widetilde{X}$ is called a covering of $X$ with projection $p: \widetilde{X} \rightarrow X$ if there is a surjection $p: V(\widetilde{X}) \rightarrow V(X)$ such that $\left.p\right|_{N(\tilde{v})}: N(\tilde{v}) \rightarrow N(v)$ is a bijection for any vertex $v \in V(X)$ and $\tilde{v} \in p^{-1}(v)$. The covering $\widetilde{X}$ is said to be regular (or $K$-covering) if there is a semiregular subgroup $K$ of $\operatorname{Aut}(\widetilde{X})$ such that the graph $X$ is isomorphic to the quotient graph $\widetilde{X} / K$, say by $h$, and the quotient map $\widetilde{X} \rightarrow \widetilde{X} / K$ is the composition $p h$ of $p$ and $h$ (in this paper all functions are composed from left to right). If the regular covering $\widetilde{X}$ is connected, then $K$ is called a covering transformation group. The fibre of an edge or a vertex is its preimage under $p$. The graph $\widetilde{X}$ is called the covering graph and $X$ is the base graph. The group of automorphisms of $\widetilde{X}$ which maps fibres to fibres is called the fibre-preserving subgroup of $\operatorname{Aut}(\widetilde{X})$.

Every edge of a graph $X$ gives rise to a pair of opposite arcs. By $e^{-1}$, we mean the reverse arc to an $\operatorname{arc} e$. Let $K$ be a finite group and denote by $A(X)$ the arc-set of $X$. An ordinary voltage assignment (or, $K$-voltage assignment) of $X$ is a function $\phi$ : $A(X) \rightarrow K$ with the property that $\phi\left(e^{-1}\right)=\phi(e)^{-1}$ for each $e \in A(X)$. The values of $\phi$ are called voltages, and $K$ is called the voltage group. The ordinary derived graph $X \times_{\phi} K$ derived from an ordinary voltage assignment $\phi: A(X) \rightarrow K$ has vertex set $V(X) \times K$ and edge set $E(X) \times K$, so that an edge $(e, g)$ of $X \times_{\phi} K$ joins a vertex $(u, g)$ to $(v, \phi(e) g)$ for $e=u v \in A(X)$ and $g \in K$. The first coordinate projection 
$p_{\phi}: X \times_{\phi} K \rightarrow X$ is a regular covering since $K$ is semiregular on $V\left(X \times_{\phi} K\right)$.

Let $p: \tilde{X} \rightarrow X$ be a $K$-covering. If $\alpha \in \operatorname{Aut}(X)$ and $\widetilde{\alpha} \in \operatorname{Aut}(\tilde{X})$ satisfy $\widetilde{\alpha} p=p \alpha$, we call $\tilde{\alpha}$ a lift of $\alpha$, and $\alpha$ the projection of $\tilde{\alpha}$. Concepts such as a lift of a subgroup of $\operatorname{Aut}(X)$ and the projection of a subgroup of $\operatorname{Aut}(\tilde{X})$ are self-explanatory. The lifts and the projections of such subgroups are of course subgroups in $\operatorname{Aut}(\tilde{X})$ and $\operatorname{Aut}(X)$, respectively. In particular, if the covering graph $\tilde{X}$ is connected, then the covering transformation group $K$ is the lift of the identity group. Gross and Tucker [11] showed that every $K$-covering of a graph $X$ can be derived from a $K$-voltage assignment which assigns the identity voltage 1 to the arcs on an arbitrary fixed spanning tree of $X$.

Let $X \times_{\phi} K \rightarrow X$ be a connected $K$-covering, where $\phi=1$ on the arcs of a spanning tree $T$ of $X$. Such $\phi$ is called a $T$-reduced voltage assignment. Then the covering graph $X \times_{\phi} K$ is connected if and only if the voltages on the cotree arcs generate the voltage group $K$.

The problem whether an automorphism $\alpha$ of $X$ lifts can be grasped in terms of voltage as follows. Observe that a voltage assignment on arcs extends to a voltage assignment on walks in a natural way. Define the mapping $\bar{\alpha}$ from the set of voltages of fundamental closed walks based at a vertex $v$ of the base graph $X$ to the voltage group $K$ as the following:

$$
(\phi(C))^{\bar{\alpha}}=\phi\left(C^{\alpha}\right)
$$

where $C$ ranges over all fundamental closed walks at $v$, and $\phi(C)$ and $\phi\left(C^{\alpha}\right)$ are the voltages of $C$ and $C^{\alpha}$, respectively. Note that if $K$ is abelian, $\bar{\alpha}$ does not depend on the choice of the base vertex, and the fundamental closed walks at $v$ can be substituted by the fundamental cycles generated by the cotree edges of $X$.

LEMMA 4.1 ([16]). Let $X \times_{\phi} K \rightarrow X$ be a connected $K$-covering. Then an automorphism $\alpha$ of $X$ lifts if and only if $\bar{\alpha}$ extends to an automorphism of $K$.

LEMMA 4.2. Let $\tilde{X}$ be a connected regular covering of the complete graph $K_{4}$, whose covering transformation group is cyclic or elementary abelian, and whose fibre-preserving subgroup is arc-transitive. Then $\tilde{X}$ is not one-regular.

Proof. Let $K$ be a cyclic or an elementary abelian group and let $\widetilde{X}=K_{4} \times_{\phi} K$ be a connected regular covering of the graph $K_{4}$ satisfying the hypotheses in the theorem, where $\phi$ is a $T$-reduced $K$-voltage assignment with the spanning tree $T$ as illustrated by dark lines in Figure 1. Identify the vertex set of $K_{4}$ with $\mathbb{Z}_{4}=\{0,1,2,3\}$ and we assign voltages $z_{1}, z_{2}$ and $z_{3}$ in $K$ to the cotree arcs as shown in Figure 1.

Suppose to the contrary that the covering graph $K_{4} \times_{\phi} K$ is one-regular. Since $K_{4}$ is not one-regular, we get $|K|>1$, and since the fibre-preserving subgroup, say $\tilde{L}$, acts arc-transitively on $K_{4} \times_{\phi} K$ and $K_{4} \times_{\phi} K$ is one-regular, we have $\operatorname{Aut}(\tilde{X})=\widetilde{L}$. 


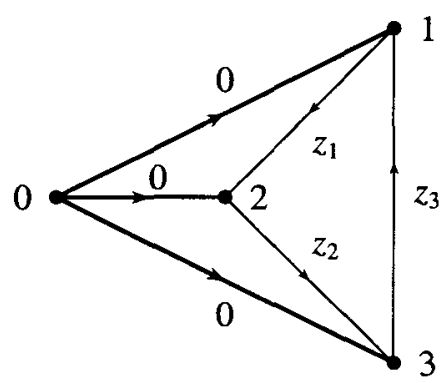

FIGURE 1. The complete graph $K_{4}$ with voltage assignment $\phi$

Hence, the projection of $\widetilde{L}$, say $L$, acts regularly on the $\operatorname{arc}$ set of $K_{4}$. Then $|L|=12$. Since $K_{4} \times_{\phi} K$ is connected, $\left\{z_{1}, z_{2}, z_{3}\right\}$ generates the voltage group $K$, that is, $\left\langle z_{1}, z_{2}, z_{3}\right\rangle=K$. Noting that $\operatorname{Aut}\left(K_{4}\right)=S_{4}$ and $|L|=12$, we have that $L=A_{4}$. Let $\alpha=(01)(23), \beta=(123)$ and $\gamma=(12)$. Clearly, $\alpha, \beta$ and $\gamma$ are automorphisms of $K_{4}$ and $\alpha, \beta \in L$.

By $i_{1} i_{2} \cdots i_{s}$, we denote a cycle which has vertex set $\left\{i_{1}, i_{2}, \ldots, i_{s}\right\}$, and edge set $\left\{\left(i_{1}, i_{2}\right),\left(i_{2}, i_{3}\right), \ldots,\left(i_{s-1}, i_{s}\right),\left(i_{s}, i_{1}\right)\right\}$. There are three fundamental cycles 012,023 and 031 in $K_{4}$, which are generated by the three cotree edges. Each cycle maps to a cycle of same length under the actions of $\alpha, \beta$ and $\gamma$. We list all these cycles and their voltages in Table 1, in which $C$ denotes a fundamental cycle of $K_{4}$ and $\phi(C)$ denotes the voltage on the cycle $C$.

Consider the mapping $\bar{\alpha}$ from the set of voltages of the three fundamental cycles of $K_{4}$ to the voltage group $K$, defined by $\phi(C)^{\bar{\alpha}}=\phi\left(C^{\alpha}\right)$, where $C$ ranges over all these three fundamental cycles. Similarly, one can define $\bar{\beta}$ and $\bar{\gamma}$. Since $L$ lifts, by Lemma $4.1 \bar{\alpha}$ and $\bar{\beta}$ can be extended to automorphisms of $K$, say $\alpha^{*}$ and $\beta^{*}$, respectively. However, $\bar{\gamma}$ can't be extended to an automorphism of $K$ because of the one-regularity of $K_{4} \times_{\phi} K$. From Table $1, z_{1}^{\beta^{*}}=z_{2}$ and $z_{2}^{\beta^{*}}=z_{3}$. This implies that $z_{1}, z_{2}$ and $z_{3}$ have the same order. Now we consider the cases according to $K$ being cyclic or elementary abelian.

Case I. $K=\mathbb{Z}_{n}(n>1)$. Since $z_{1}, z_{2}$ and $z_{3}$ have the same order and $\left\langle z_{1}, z_{2}, z_{3}\right\rangle=\mathbb{Z}_{n}$, each of them generates the cyclic group $\mathbb{Z}_{n}$. Thus we may assume that $z_{1}=1$. Let $1^{\beta^{*}}=k$. Then $(k, n)=1$. By $z_{1}^{\beta^{*}}=z_{2}, z_{2}^{\beta^{*}}=z_{3}$ and $z_{3}^{\beta^{*}}=z_{1}$ (see Table 1), we have that $z_{2} \equiv k(\bmod n), z_{3} \equiv k^{2}(\bmod n)$ and $k^{3} \equiv 1(\bmod n)$. Let $1^{\alpha^{*}}=l$. Then $z_{1}^{\alpha^{*}}=z_{3}$ and $z_{3}^{\alpha^{*}}=z_{1}$ implies that $l \equiv k^{2}(\bmod n)$ and $l k^{2} \equiv 1(\bmod n)$. From the latter equation and $k^{3} \equiv 1(\bmod n)$, we have $k \equiv l(\bmod n)$. Thus $l \equiv k^{2}(\bmod n)$ implies that $k \equiv 1(\bmod n)$ because $(k, n)=1$. It follows that $z_{1} \equiv z_{2}(\bmod n) \equiv z_{3}$ $(\bmod n) \equiv 1(\bmod n)$ and so $\bar{\gamma}$ can be extended to an automorphism of $\mathbb{Z}_{n}$ induced by $1 \mapsto-1$, a contradiction.

Case II. $K=\mathbb{Z}_{p} \times \mathbb{Z}_{p} \times \cdots \times \mathbb{Z}_{p}=\mathbb{Z}_{p}^{m}(p$ prime, $m \geq 2)$. By $\left\langle z_{1}, z_{2}, z_{3}\right\rangle=\mathbb{Z}_{p}^{m}$, we 
TABLE 1. Fundamental cycles and their images with corresponding voltages on $K_{4}$

\begin{tabular}{|c|c||c|c||c|c||c|c|}
\hline$C$ & $\phi(C)$ & $C^{\alpha}$ & $\phi\left(C^{\alpha}\right)$ & $C^{\beta}$ & $\phi\left(C^{\beta}\right)$ & $C^{\gamma}$ & $\phi\left(C^{\gamma}\right)$ \\
\hline 012 & $z_{1}$ & 103 & $z_{3}$ & 023 & $z_{2}$ & 021 & $-z_{1}$ \\
\hline 023 & $z_{2}$ & 132 & $-z_{1}-z_{2}-z_{3}$ & 031 & $z_{3}$ & 013 & $-z_{3}$ \\
\hline 031 & $z_{3}$ & 120 & $z_{1}$ & 012 & $z_{1}$ & 032 & $-z_{2}$ \\
\hline
\end{tabular}

may assume that $K=\mathbb{Z}_{p}^{2}$ or $\mathbb{Z}_{p}^{3}$. If $K=\mathbb{Z}_{p}^{3}$, then $z_{1}, z_{2}$ and $z_{3}$ are linearly independent. Similarly, $-z_{1},-z_{2}$ and $-z_{3}$ are also linearly independent. This implies that $\bar{\gamma}$ can be extended to an automorphism of $\mathbb{Z}_{p}^{3}$, a contradiction.

Now suppose that $K=\mathbb{Z}_{p}^{2}=\langle a\rangle \times\langle b\rangle$. By $z_{1}^{\beta^{*}}=z_{2}, z_{2}^{\beta^{*}}=z_{3}$ and $z_{3}^{\beta^{*}}=z_{1}, z_{1}$ and $z_{2}$ must be linearly independent. We may assume that $z_{1}=a$ and $z_{2}=b$. Let $z_{3}=$ $k a+l b=z_{2}^{\beta^{*}}$. Then $z_{3}^{\beta^{*}}=z_{1}$ implies that $l k \equiv 1(\bmod p)$ and $k+l^{2} \equiv 0(\bmod p)$, and by $l k \equiv 1(\bmod p)$ we have $(l, p)=1$. Since $z_{1}^{\alpha^{*}}=z_{3}$ and $z_{2}^{\alpha^{*}}=-z_{1}-z_{2}-z_{3}$ means that $a^{\alpha^{*}}=k a+l b$ and $b^{\alpha^{*}}=-(k+1) a-(l+1) b$, we may deduce that $a=\left(k^{2}-l k-l\right) a+l(k-l-1) b$ from $z_{3}^{\alpha^{*}}=z_{1}$, in which $b$ has the coefficient $l(k-l-1)$. Since $(l, p)=1, k-l-1 \equiv 0(\bmod p)$. Noting that $l k \equiv 1(\bmod p)$ and $k+l^{2} \equiv 0(\bmod p)$ we have $l^{2}+l+1 \equiv l^{2}+l-1(\bmod p) \equiv 0(\bmod p)$, implying that $p=2$. This is impossible because the equation $l^{2}+l+1 \equiv 0(\bmod 2)$ has no solution.

THEOREM 4.3. Let $p$ be a prime. Then there is no one-regular cubic graph of order $4 p$ or $4 p^{2}$.

PROOF. By Proposition 2.8 we may assume that $p \geq 17$ and suppose to the contrary that $X$ is a one-regular cubic graph of order $4 p$ or $4 p^{2}$. Since $X$ is connected, $A=\operatorname{Aut}(X)$ is transitive on $V(X)$. By the one-regularity of $X,|A|=12 p$ or $12 p^{2}$. By [10, pp. 12-14], a non-abelian simple $\{2,3, p\}$-group is one of the following groups: $A_{5}, A_{6}, L_{2}(7), L_{2}(8), L_{2}(17), L_{3}(3), U_{3}(3)$, and $U_{4}(2)$. By Conway et al. [5], the orders of these simple groups have divisor 8 or 9 except $A_{5}$. Since $|A|$ has no divisor 8 or 9 and $p \geq 17, A$ is solvable. Let $\pi=\{2, p\}$. By Proposition $2.4, A$ has a Hall $\pi$-subgroup, say $H$.

We claim that $A$ has a normal Sylow $p$-subgroup. Consider the conjugate action of $A$ on the set of cosets of $H$ in $A$. Then $A / H_{A}$ is isomorphic to a subgroup of the symmetric group $S_{3}$ of degree 3 , where $H_{A}$ is the largest normal subgroup of $A$ contained in $H$. Since $H_{A} \leq H$ and $|A: H|=3$, we have $\left|A / H_{A}\right|=3$ or 6 . If $\left|A / H_{A}\right|=3$ then $H_{A}=H$, and if $\left|A / H_{A}\right|=6$ then $\left|H: H_{A}\right|=2$. Thus $\left|H_{A}\right|=2 p$, $4 p, 2 p^{2}$ or $4 p^{2}$. By Sylow's theorem, the Sylow $p$-subgroup of $H_{A}$ is normal in $H_{A}$ and so is normal in $A$. Since Sylow $p$-subgroups of $H_{A}$ are also Sylow $p$-subgroups of $A, A$ has a normal Sylow $p$-subgroup. 
Let $N$ be the normal Sylow $p$-subgroup of $A$. Since $|N|$ has no divisor $3, N$ acts semiregular on $V(X)$. It follows that $N$ has four orbits. Recall that $\bar{X}$ is the quotient graph of $X$ corresponding to the orbits of $N$, where $\bar{X}$ has the same definition as in the proof of Lemma 3.2. Then $\bar{X}$ is isomorphic to $K_{4}$, and hence $X$ is a regular covering of $K_{4}$ with the covering transformation group $N$ and with the fibre-preserving subgroup Aut $(X)$. Since $|N|=p$ or $p^{2}, N$ is cyclic or elementary abelian and by Lemma 4.2 $X$ can't be one-regular, a contradiction.

\section{References}

[1] B. Alspach, D. Marušič and L. Nowitz, 'Constructing graphs which are $\frac{1}{2}$-transitive', J. Austral. Math. Soc. (A) 56 (1994), 391-402.

[2] Y. Cheng and J. Oxley, 'On weakly symmetric graphs of order twice a prime', J. Combin. Theory (B) 42 (1987), 196-211.

[3] M. D. E. Conder and P. Dobcsányi, 'Trivalent symmetric graphs on up to 768 vertices', J. Combin. Math. Combin. Comput. 40 (2002), 41-63.

[4] M. D. E. Conder and C. E. Praeger, 'Remarks on path-transitivity on finite graphs', Europ. J. Combin. 17 (1996), 371-378.

[5] J. H. Conway, R. T. Curtis, S. P. Norton, R. A. Parker and R. A. Wilson, Atlas of finite groups (Oxford University Press, Oxford, 1985).

[6] D. Ž. Djoković and G. L. Miller, 'Regular groups of automorphisms of cubic graphs', J. Combin. Theory $(B) 29$ (1980), 195-230.

[7] X. G. Fang, J. Wang and M. Y. Xu, 'On 1-arc-regular graphs', Europ. J. Combin. 23 (2002), $785-791$.

[8] Y. Q. Feng, J. H. Kwak and M. Y. Xu, 's-regular cubic Cayley graphs on abelian or dihedral groups', Research Report No. 53, (Institute of Math. and School of Math. Sci, Peking Univ., 2000).

[9] R. Frucht, 'A one-regular graph of degree three', Canad. J. Math. 4 (1952), 240-247.

[10] D. Gorenstein, Finite simple groups (Plenum Press, New York, 1982).

[11] J. L. Gross and T. W. Tucker, 'Generating all graph coverings by permutation voltage assignment', Discrete Math. 18 (1977), 273-283.

[12] F. Harary, Graph theory (Addison-Wesley, Reading, MA, 1969).

[13] B. Huppert, Endliche Gruppen I (Springer, Berlin, 1967).

[14] P. Lorimer, 'Vertex-transitive graphs: symmetric graphs of prime valency', J. Graph Theory 8 (1984), 55-68.

[15] A. Malnič, D. Marušič and N. Seifter, 'Constructing infinite one-regular graphs', Europ. J. Combin. 20 (1999), 845-853.

[16] A. Malnič, R. Nedela and M. Škoviera, 'Lifting graph automorphisms by voltage assignments', Europ. J. Combin. 21 (2000), 924-947.

[17] D. Marušic, 'A family of one-regular graphs of valency 4', Europ. J. Combin. 18 (1997), 59-64.

[18] D. Marušič and R. Nedela, 'Maps and half-transitive graphs of valency 4', Europ. J. Combin. 19 (1998), 345-354.

[19] D. Marušič and M. Y. Xu, 'A $\frac{1}{2}$-transitive graph of valency 4 with a nonsolvable group of automorphisms', J. Graph Theory 25 (1997), 133-138. 
[20] R. C. Miller, 'The trivalent symmetric graphs of girth at most six', J. Combin. Theory (B) 10 (1971), 163-182.

[21] C. E. Praeger, 'Imprimitive symmetric graphs', Ars Combinatoria 19A (1985), 149-163.

[22] D. J. Robinson, A course in the theory of groups (Springer, New York, 1982).

[23] H. Wielandt, Finite permutation groups (Academic Press, New York, 1964).

[24] M. Y. Xu, 'A note on one-regular graphs', Chinese Sci. Bull. 45 (2000), 2160-2162.

Department of Mathematics

Beijing Jiaotong University

Beijing 100044

P.R. China

e-mail: yqfeng@center.njtu.edu.cn
Department of Mathematics

Pohang University of Science

and Technology

Pohang

790-784 Korea

e-mail: jinkwak@postech.ac.kr 